\title{
TEMPORAL VARIATION GOVERNS THE PROLIFERATION OF DIFFERENT TAXA OR GUILDS OF ULTRAPLANKTONIC FUNGI IN A LARGE RESERVOIR
}

\author{
Hou, Y. Y. ${ }^{1 \#}-$ LIU, K. S. ${ }^{1 \#}-$ LIU, X. X. ${ }^{1}-$ HoU, J. J. ${ }^{1}-$ LI, Y. T. ${ }^{1,2^{*}}-$ BI, Y. H. ${ }^{3 *}$ \\ ${ }^{1}$ Hubei Key Laboratory of Edible Wild Plants Conservation and Utilization, Hubei Normal \\ University, Huangshi 435002, China \\ ${ }^{2}$ Bengbu University, Bengbu 233030, China \\ ${ }^{3}$ State Key Laboratory of Freshwater Ecology and Biotechnology, Institute of Hydrobiology, \\ The Chinese Academy of Sciences, Wuhan 430072, China \\ ${ }^{\#}$ These two authors made equal contributions to this paper \\ *Corresponding authors \\ e-mail/phone: liyuntao@hbnu.edu.cn/+86-714-651-1613; biyh@ihb.ac/+86-276-878-0016
}

The two corresponding authors made equal contributions to this paper

(Received $9^{\text {th }}$ Mar 2020; accepted $29^{\text {th }}$ Jul 2020)

\begin{abstract}
The fungus play an important role within the aquatic ecosystem. However, the ultraplanktonic fungus diversity in reservoir ecosystems is still greatly unexplored. In this study, we used Illumina sequencing technology to analyze the diversity of ultraplanktonic fungi (size class $0.7-5 \mu \mathrm{m}$ in this study) from two sampling sites along the Three Gorges Reservoir of the Yangtze River in China at four seasons in 2015. Basidiomycota was the most abundant phylum (36.69\% of the total fungal reads), followed by Ascomycota (27.05\%), Cryptomycota (18.06\%), Chytridiomycota, $(15.81 \%)$ and Blastocladiomycota $(1.51 \%)$. Our study showed that ultraplanktonic fungi were changing with time, and a shift in dominant taxonomic groups was observed between time points. A marked temporal variation in functional guilds of fungi was also observed, suggesting that temporal variation governs the proliferation of different taxa or guilds of fungi in the reservoir. Our results suggest that inputs from fungal species of terrestrial origin may be responsible for the observed changes in pelagic community composition. This result also implies that terrestrial inputs may be an important source of fungal species in reservoir ecosystems. Additionally, it is possible that the chytrids were limited by hyperparasitic Cryptomycota.
\end{abstract}

Keywords: reservoir ecosystem, aquatic fungi, high-throughput sequencing, diversity and distribution, functional guilds

\section{Introduction}

Fungi represent one of the last frontiers of the unexplored biodiversity that challenges microbial ecology today (Lepère et al., 2018). The kingdom of fungi is highly diverse. Recent estimates indicate that the number of aquatic fungal species lies between 3000 and 4000 (Grossart and Rojas-Jimenez, 2016). Aquatic fungi have been detected from a wide range of habitats, including the ocean (Pachiadaki et al., 2016; Hassett et al., 2019), coastal regions (Gao et al., 2010; Duan et al., 2018), lakes (Lefèvre et al., 2012; Comeau et al., 2016; Wahl et al., 2018), and rivers (Duarte et al., 2015; Seena et al., 2019). However, relatively few studies have investigated the biodiversity and ecological role of mycoplankton in reservoir ecosystems.

Fungi play an important role in aquatic ecosystems. They influence and shape them by cycling organic matter and channeling nutrients across trophic levels (Nilsson et al., 
2019). Some fungi decompose leaves and wood (Canhoto et al., 2017), and others are important sources of food and nutrients for filter-feeding zooplankton and copepods (Rasconi et al., 2011; Sime-Ngando et al., 2011; Frenken et al., 2018). Moreover, rather than just serving as prey and a source of nutrients, fungi have been found to potentially establish alternative trophic links between large inedible phytoplankton and zooplankton through the "mycoloop" pathway (Kagami et al., 2014; Agha et al., 2016; Gerphagnon et al., 2019).

The majority of aquatic fungi occur in freshwaters (Shearer et al., 2007). As threats to freshwater biodiversity escalate, there is an urgent need to survey, collect and isolate freshwater fungal species. Most aquatic fungi are microscopic (Wurzbacher et al., 2011), hence ultraplanktonic fungi have a major role in aquatic environments. Despite their ecological importance, ultraplanktonic fungi have remained poorly described because of their small size and lack of morphological characteristics. Therefore, it is important to understand ultraplanktonic fungi diversity in freshwater ecosystems. In some studies, culture-independent molecular techniques have been exclusively used to determine fungal diversity in freshwater habitats. Several studies have surveyed the composition, distribution, and function of planktonic fungi in reservoir ecosystems (Chen et al., 2018). Unfortunately, most studies of large freshwater systems are conducted at one time points. For diversity studies, the inclusion of time surveys allows for the detection of a wider diversity than time-point studies (Simon et al., 2015). Nevertheless, knowledge is scarce regarding temporal series diversity of ultraplanktonic fungi in large reservoir ecosystems. Lack of information on ultraplanktonic fungi in large reservoir ecosystems limits the comprehensive understanding of aquatic ecosystems.

The Three Gorges Reservoir is located in the upstream reaches of Yangtze River in China. Presently, it is one of the largest reservoirs in the world (Han et al., 2015). In this study, a high-throughput Illumina sequencing technology was used to analyze the diversity of ultraplanktonic fungi (size class $0.7-5 \mu \mathrm{m}$ in this study). Two sites were considered along the Three Gorges Reservoir of the Yangtze River and sampled over four seasons in 2015. The objective of our study was to provide a taxonomic and functional characterization of the ultraplanktonic fungal community of a large reservoir at two diametrically opposed sites on 4 occasions during the year.

\section{Materials and methods}

\section{Sampling and measurement of physico-chemical parameters}

In total, eight water samples were collected at two sites (CJ01 and CJ05) from the Three Gorges Reservoir of the Yangtze River of China in the following months of 2015: April (spring), August (summer), October (autumn), and December (winter) (Fig. 1). The Three Gorges Reservoir is a canyon-shaped, typical river-type reservoir. The water body type of the two sample sites were the same. These sites are in a transition-type region (Zheng et al., 2006). The sampling sites were located in the middle of the river; CJ01 (E 110.99990, N 30.82111) is approximately $1.6 \mathrm{~km}$ and CJ05 (E 110.84682, N 30.88436) is approximately $200 \mathrm{~m}$ away from the riverbank. The sampling site CJ01 was located approximately $1 \mathrm{~km}$ upstream of the Three Gorges Dam.

Water samples were collected from a depth of about $0.5 \mathrm{~m}$ beneath the water surface by using a 5 -L sampler. These samples were pre-filtered through 5 - $\mu \mathrm{m}$ pore-size polycarbonate filters (Millipore, USA). Then, these samples were filtered through GF/F 
filters $(0.7 \mu \mathrm{m}$, Whatman $)$. The filters were immediately frozen in liquid nitrogen and stored at $-80{ }^{\circ} \mathrm{C}$ until further analysis.

Physico-chemical factors, such as water temperature (WT), dissolved oxygen (DO), $\mathrm{pH}$, and turbidity, were measured in situ with an YSI model Professional Plus multiparameter probe (YSI, USA). According to standard methods described in previous studies, we determined the following parameters: chemical oxygen demand (COD), oxidation-reduction potential (ORP), the concentrations of total nitrogen (TN), orthophosphate $\left(\mathrm{PO}_{4}{ }^{3-}\right)$, nitrate $\left(\mathrm{NO}_{3}{ }^{-}\right)$, total phosphorus (TP), and ammonium $\left(\mathrm{NH}_{4}{ }^{+}\right)$ (Huang et al., 1999). Trophic states of different sampling sites and times were determined from comprehensive nutritional status index (trophic level index, TLI) by using the values of following parameters: TN, TP, and COD (Tian et al., 2017). Trophic states were set as follows: $\mathrm{TLI} \leq 30$, oligotrophic; $30<\mathrm{TLI} \leq 50$, mesotrophic; $50<\mathrm{TLI} \leq 60$, slightly eutrophic; $60<\mathrm{TLI} \leq 70$, moderately eutrophic; $\mathrm{TLI}>70$, highly eutrophic (Meng and Zhao, 2007).

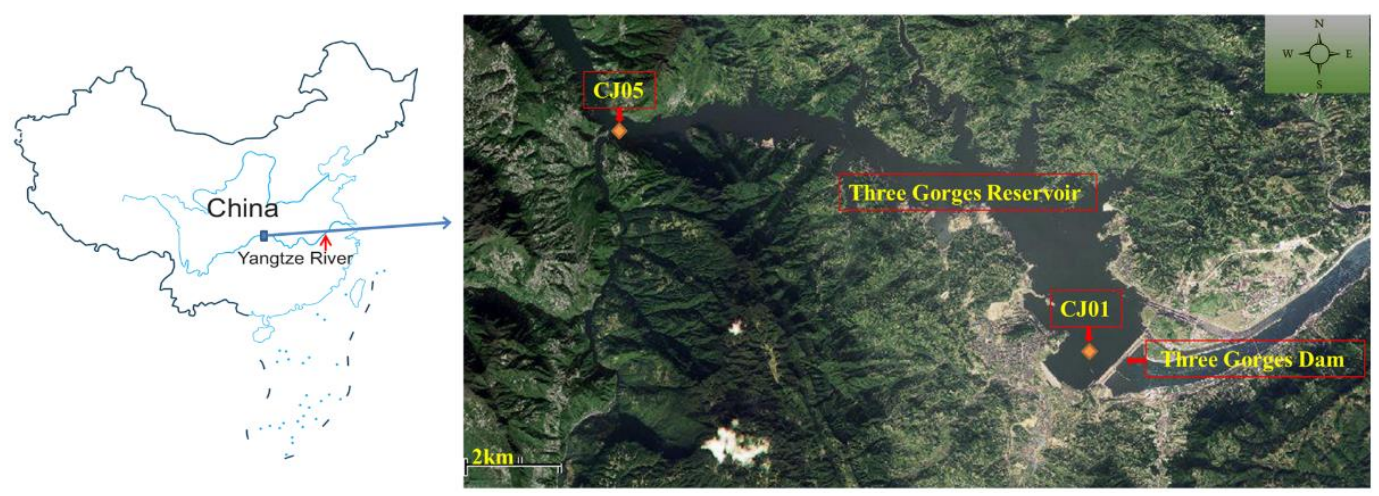

Figure 1. Map of the study area and sampling site

\section{DNA extraction, amplification, and sequencing of $18 S$ rDNA fragments}

A previously described method was used to extract DNA from filters (Countway et al., 2005). To amplify the $18 \mathrm{~S}$ small subunit rRNA gene fragments from samples, we used the following fungal-specific primers: $817 \mathrm{~F}$ (5'TTAGCATGGAATAATRRAATAGGA $\left.-3^{\prime}\right)$ and 1196R (5'CCTTTGAGTGGTCCAGGTCT -3') (Borneman and Hartin, 2000). To modify primer sequences, Illumina adaptor was added to the ends of the forward primer and another adaptor was added to the ends of the reverse primer. After amplification, the amplicons were purified directly by using UNIQ-10 PCR purification kit (Sangon Biotech Ltd, Shanghai, China); the purified amplicons were quantified with TBS-380 (Turner BioSystems Inc, USA). Paired-end sequencing of purified amplicons was carried out on an Illumina MiSeq PE250 platform (Majorbio Bio-Pharm Technology Ltd, Shanghai, China).

\section{Sequence processing}

The raw demultiplexed reads were first quality trimmed Mothur software version 1.28.0 (Schloss et al., 2009). Following these filtering parameters, some reads were removed due to quality considerations: the length of nucleotides was less than $300 \mathrm{bp}$; it contained ambiguous bases, which were imperfectly matched with a sample-specific 
barcode; and the average sequencing quality scores were less than 25 for the whole sequence. Potentially chimeric sequences were identified and removed using UCHIME (Edgar, et al., 2011). By using the furthest neighbour method (http://www.mothur.org/wiki/Cluster), we clustered unique sequences into operational taxonomic units (OTUs) at $97 \%$ sequence identity. Subsequently, a representative sequence from each OTU was aligned against the SILVA 123 reference alignment using the RDP classifier (Wang et al., 2007). The functional group (guild) of fungal OTUs was determined by using FUNGuild v1.0 database (Nguyen et al., 2016).

\section{Statistical analyses}

Diversity indices (Shannon-Wiener and Pielou's evenness index) of each sample were calculated by using the $\mathrm{R}$ (Ihaka and Gentleman, 1996) package vegan (Oksanen et al., 2016). Bray-Curtis distances were used to compare community structures with non-metric multidimensional scaling (NMDS). The significance of observed differences in community structure was determined by permutational multivariate analysis of variance (PERMANOVA), which is based on Bray-Curtis distance using 9999 permutations. Similarity percentage analysis (SIMPER) was used to identify which OTUs contribute the most for observable difference between groups. Moreover, NMDS, PERMANOVA and SIMPER were carried out using $\mathrm{R}$ package vegan. The non-parametric Mann-Whitney (two groups) and Kruskal-Wallis tests (more groups) were employed to evaluate differences. These tests were performed by using SPSS software version 24 (IBM, USA). R pheatmap package (Kolde, 2019) was used to construct a heatmap of the top 10 most abundant OTUs. We identified the OTUs that differed significantly $(q<0.05)$ for two time points with White's non-parametric t-test and Storey's multiple testing correction; the Statistical Analyses of Metagenomic Profiles (STAMP) software version 2.1 was used to perform these tests (Parks et al., 2014).

To determine the correlation between ultraplanktonic fungi communities and environmental factors, canonical-correlation analysis (CCA), was performed using $\mathrm{R}$ package vegan. The CCA was performed using the relative abundance of reads data for the fungal phyla and all the physico-chemical factors. To identify environmental factors that significantly correlate with ultraplanktonic fungi communities, an alternative CCA model was constructed using the step function in $\mathrm{R}$ and Akaike's information criterion (AIC).

\section{Nucleic acid sequence accession numbers}

In this study, the raw sequencing data were submitted to the Sequence Read Archive (SRA) of NCBI (National Center for Biotechnology Information) (Accession number SAMN06107068).

\section{Results}

\section{Characterization of environmental variables}

In this study, we measured 11 physico-chemical factors at the two sampling sites: CJ01 and CJ05 (Table Al). No significant differences were found in the physicophysical-chemical factors between the two sampling sites ( $p>0.3$, MannWhitney U-test). Moreover, these factors did not significantly differ across the four time 
points (all $p>0.08$, Kruskal-Wallis test). The whole trophic state of Three Gorges Reservoir is mesotrophic, except CJ05 site, which was slightly eutrophic in summer $(\mathrm{TLI}=51.4)$. At the CJ01 and CJ05 sampling sites, the trophic state did not differ significantly (Mann-Whitney U-test, $p=1.0$ ); however, the TLI indexes of CJ05 site were greater than that of CJ01 site (except in summer) (Table Al).

\section{Overall community composition of ultraplanktonic fungi}

In this study, 283,191 reads (most between 390 and 400 bp) were obtained after removing denoising and chimera. After plotting rarefaction curves of OTU richness for each sample, we inferred that total diversity could be recovered from all the samples (Fig. A1). We obtained a total of 35,393 high-quality fungal reads, ranging from 1923 to 7444 per sample. All reads were grouped into 192 OTUs by using a cut-off value of $97 \%$ sequence identity. Of the 192 OTUs, 61 OTUs belong to fungi. These fungi OTUs ranging from 28 to 46 OTUs per sample (Table 1). The other 131 OTUs mainly affiliated to algae and protozoan. Only three and seven fungal OTUs were unique for CJ01 and CJ05 sites, respectively (Fig. A2).

Table 1. Summary of the diversity index of each sample

\begin{tabular}{c|c|c|c|c|c}
\hline Season & Sample sites & Reads & OTUs & Shannon-Wiener & Pielou evenness \\
\hline \multirow{2}{*}{ Spring } & CJ01 & 1923 & 32 & 1.454 & 0.419 \\
& CJ05 & 3140 & 28 & 1.442 & 0.428 \\
\hline \multirow{2}{*}{ Summer } & CJ01 & 3826 & 43 & 3.165 & 0.831 \\
& CJ05 & 6679 & 46 & 2.941 & 0.764 \\
\hline \multirow{2}{*}{ Autumn } & CJ01 & 6529 & 44 & 2.397 & 0.63 \\
& CJ05 & 7444 & 41 & 2.522 & 0.671 \\
\hline \multirow{2}{*}{ Winter } & CJ01 & 3526 & 28 & 2.517 & 0.755 \\
& CJ05 & 2326 & 30 & 2.281 & 0.671 \\
\hline
\end{tabular}

OTU: operational taxonomic unit

Fungal reads were assigned to the following five fungal phyla: Basidiomycota, Ascomycota, Chytridiomycota, Cryptomycota, and Blastocladiomycota (Fig. 2A). Furthermore, there was the group fungi_LKM15, which named after the first clone encountered in an environmental DNA survey in Lake Ketelmeer and referred to some undescribed fungi (van Hannen et al., 1999). The classification of 61 fungal OTUs is as follows: nineteen fungal OTUs belonged to Ascomycota (27.05\% of total fungi reads); eighteen fungal OTUs belonged to Basidiomycota (36.69\% of the reads); ten fungal OTUs belonged to Chytridiomycota ( $15.81 \%$ of the reads); six fungal OTUs belonged to Cryptomycota (18.06\% of the reads); one fungal OTU belonged to Blastocladiomycota ( $1.51 \%$ of the reads); two fungal OTUs belong to LKM15 group ( $0.35 \%$ of the reads); and five fungal OTUs were considered as unknown fungi ( $0.53 \%$ of the reads). The 10 most frequently observed OTUs constituted $74.07 \%$ of total fungal reads, these OTUs belong to Ascomycota, Basidiomycota, Chytridiomycota, and Cryptomycota (Fig. 2B).

By matching sequences with Ascomycota, we observed that there was high affinity with four known classes. Most Ascomycota reads were affiliated with Dothideomycetes (15.69\% of the total fungal reads). Meanwhile, Eurotiomycetes and Sordariomycetes constituted $3.59 \%$ and $4.23 \%$ of the total fungal reads, respectively. The most common 
Basidiomycota classes were as follows: the Microbotryomycetes $(15.51 \%$ of the total fungal reads), Exobasidiomycetes (9.08\%), and Agaricomycetes (8.03\%). Cryptomycota was mostly composed of incertae sedis taxa. The diversity of most Chytridiomycota was represented by Zygorhizidium (74.84\% of the total Chytridiomycota reads).

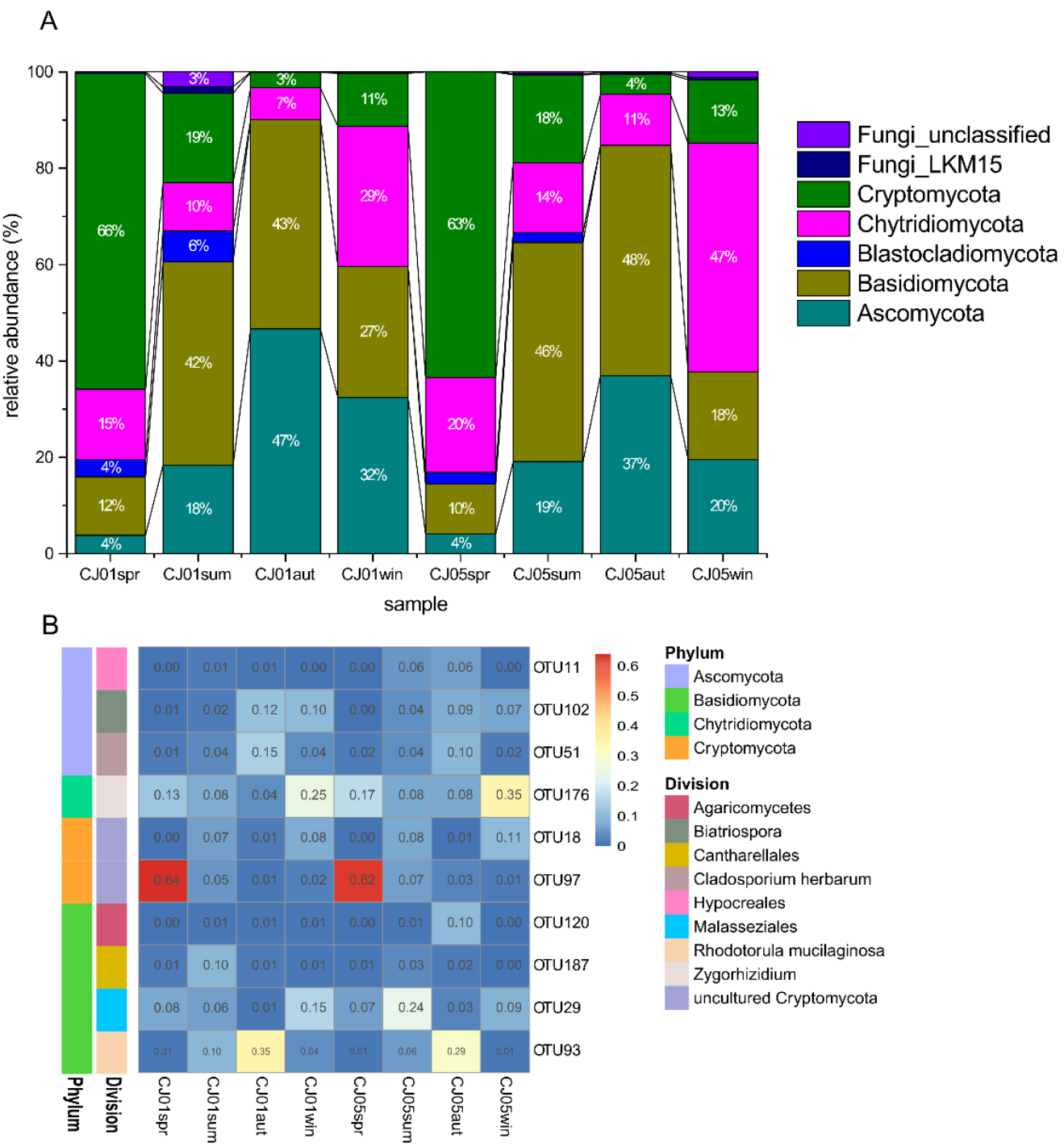

Figure 2. Variation in fungal community compositions at each sampling site within one year. (A) Taxonomic composition at the phylum level. (B) Heat map showing the relative abundance of the top 10 most abundant operational taxonomic units (OTUs) among the 61 fungal OUTs. Spr: spring, sum: summer, aut: autumn, win: winter

Only 30 fungal OTUs were assigned to a functional group by FUNGuild database. In this study, six trophic modes and eleven functional guilds were found (Fig. 3). Saprotrophs occupied most of the OTUs (14 OTUs); pathotroph-saprotroph possessed most reads $(15.52 \%$ of total fungal reads) despite containing only one OTU. Saprotroph-symbiotroph occupied one OTU and least reads $(2.6 \%$ of the total fungal reads). Among the 11 functional guilds, undefined saprotroph had the largest percentage of assigned OTUs (12 OTUs). Plant pathogen (six OTUs) and fungal parasite-undefined 
saprotroph (four OTUs) were the other moderately abundant guilds. Animal Endosymbiont-Undefined Saprotrophs comprised of one OTU but most percentages of fungal reads (15.52\% of total fungal reads).
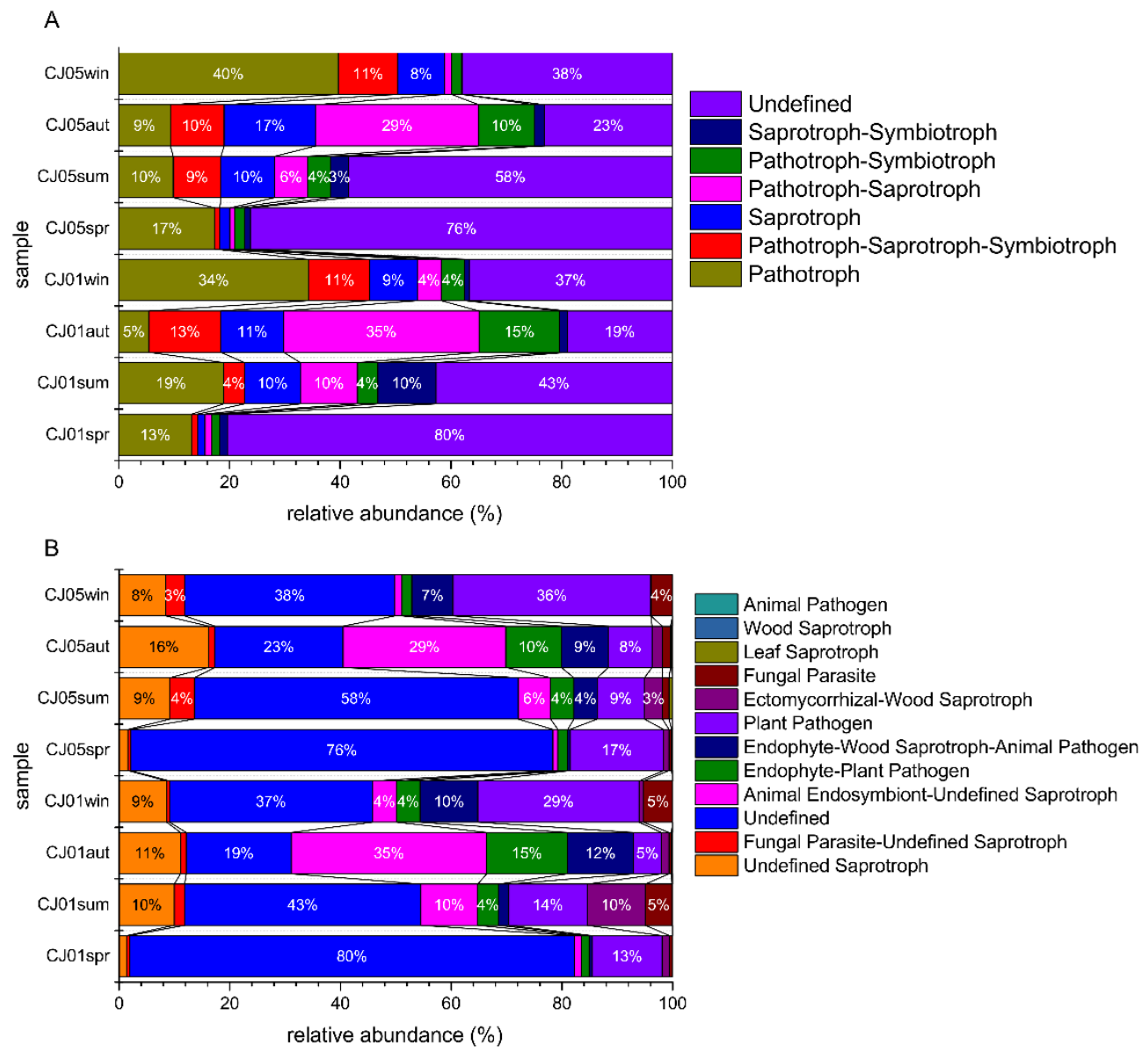

Figure 3. Compositions of fungal functional guild and trophic mode inferred by FUNGuild. (A) Functional guild. (B) Trophic mode

Basidiomycota, Ascomycota, and Chytridiomycota were assigned to nine, four, and two functional guilds, respectively. Basidiomycota was assigned to five trophic modes, while Ascomycota was assigned to four trophic modes. The trophic modes of Chytridiomycota were pathotroph (two OTUs) and saprotroph (one OTU).

\section{Variations of community structure and composition}

The diversity indices (Shannon-Wiener and Pielou's evenness) did not differ significantly between the two sampling sites: CJ01 and CJ05 sites (all $p>0.8$, MannWhitney U-test). Moreover, the diversity indices did not change significantly among the four time points (Kruskal-Wallis test, $p>0.09$ ). Fungal communities were primarily differentiated by time (Fig. 4) and differences in community structure of ultraplanktonic fungi were significant (PERMANOVA, $p=0.0101$ ); no significant difference were found between sampling sites (PERMANOVA, $p=0.8278$ ). Based on the overall fungal community composition (at the OTU resolution), SIMPER analysis indicate that 
temporal differences were mainly associated with the following OTUs: OTU97 (Cryptomycota, 23.94\%), OTU93 (Basidiomycota, Rhodotorula, 15.94\%), OTU29 (Basidiomycota, Malasseziales, 7.196\%), OTU51 (Ascomycota, Cladosporium, 5.94\%), OTU102 (Ascomycota, Biatriospora, 5.52\%) and OTU176 (Chytridiomycota, Zygorhizidium, 5.28\%).

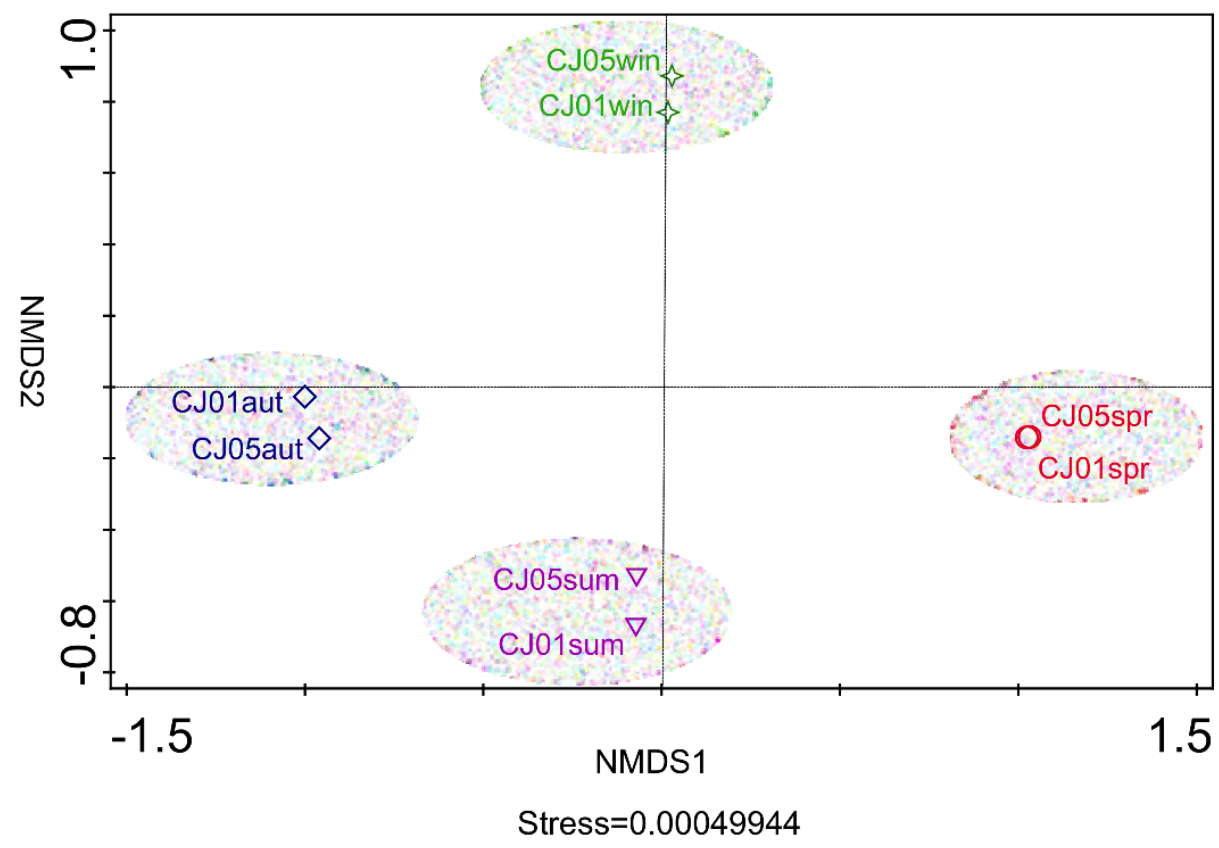

Figure 4. Non-metric multidimensional scaling (NMDS) plot based on Bray-Curtis distances

In this study, dominant phyla varied with time (Fig. 2A). Cryptomycota and Chytridiomycota were dominant in spring and winter seasons, respectively. In warm seasons (summer and autumn), Basidiomycota and Ascomycota dominated total fungal reads. Most of the top 10 abundant OTUs showed temporal variations (Fig. 2B). Seven of the top 10 abundant OTUs achieved maximum abundance in the warmer season, while the other OTUs showed maximum abundance in winter or spring season. For example, OTU176 (Chytridiomycota, Zygorhizidium) was present in all seasons; however, the abundance of OTU176 was maximum in winter. Moreover, OTU93 (Basidiomycota, Rhodotorula) was predominant in autumn. Furthermore, OTU97 (Cryptomycota) accounted for over $60 \%$ of the fungal reads in spring. We found that the relative abundance of three of the 61 OTUs varied significantly between two time points (Fig. 5). Compared to summer, the relative abundance of OTU97 (Cryptomycota) was significantly higher in spring (White's non-parametric t-test, $q<0.01$ ). Moreover, OTU86 (Ascomycota, Trematosphaeria) was more abundant in autumn than in summer (White's non-parametric t-test, $q<0.01$ ). Furthermore, OTU145 (Basidiomycota, Phallus) was far more abundant in autumn than in winter (White's non-parametric ttest, $q<0.01$ ).

Individual OTUs showed variable patterns of temporal change on a finer scale (Fig. 6). The read numbers of most OTUs were more abundant in a particular season. Temporal patterns were observed in the relative abundance of these OTUs. Few OTUs showed consistent abundance, regardless of season (for example, OTU 176, 
Chytridiomycota). Some OTUs had a relatively low abundance in most seasons; however, they showed a relatively high relative abundance in a certain season (OTU 93, Basidiomycota, showed relatively higher abundance in autumn; OTU 97 and OTU 189, Cryptomycota, showed relatively higher abundance in winter) or in some seasons (OTU 18, Basidiomycota were relatively abundant in following seasons: summer and winter). Furthermore, several OTUs were found consistently in a certain season (OTU 86, Ascomycota was found consistently in autumn).

By performing a comparative analysis of the defined functional guild structures in the four time points, we observed a distinct distribution. In the CJ01 site, endophyteplant pathogen accounted for $15 \%$ and $1.46 \%$ of fungal reads in autumn and spring, respectively (Fig. 3). The trophic mode of pathotroph-saprotroph peaked to $35 \%$ in autumn, but it was very low in spring. The percentage of some defined functional guilds, such as undefined saprotroph, was relatively higher in autumn. $(11 \%$ and $16 \%$ in CJ01 and CJ05 sampling sites, respectively) (Fig. 3A).
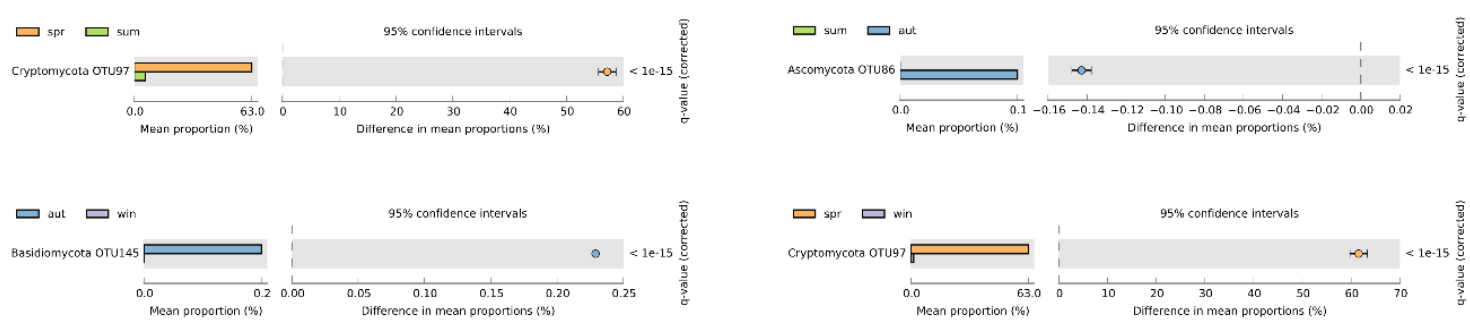

Figure 5. OTUs that were significantly different between two seasons
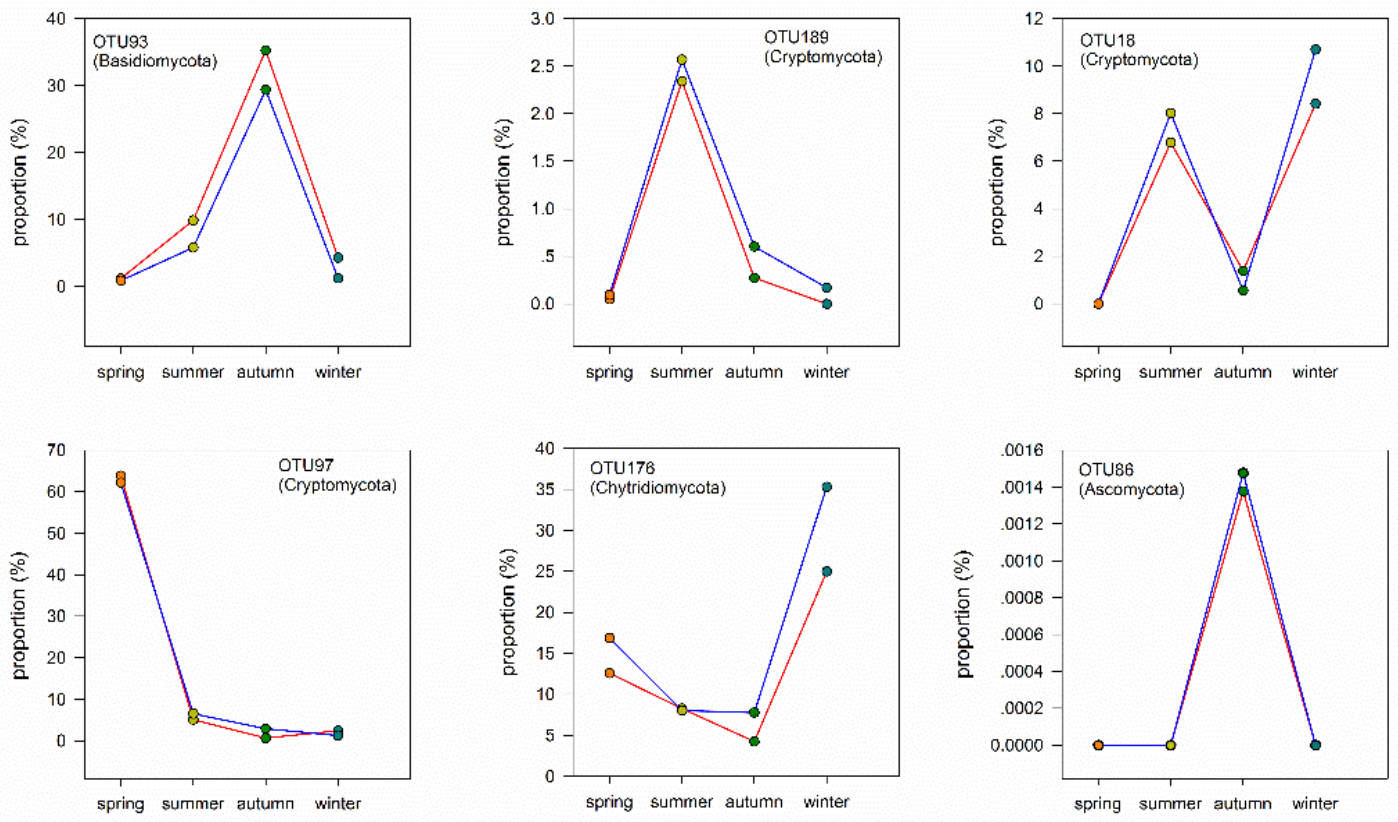

$$
\rightarrow \text { CJ01 }
$$

Figure 6. Relative abundances of six fungal OTUs showing different types of dynamics 


\section{Correlation of ultraplanktonic fungi and physico-chemical parameters}

CCA analysis was conducted for five phyla and three (from 11) forward selected environmental variables (WT, $\mathrm{pH}$, and $\mathrm{PO}_{4}{ }^{3-}$ ) (Fig. 7). Environmental variables accounted for $91.42 \%$ of the variance associated with ultraplanktonic fungi community structure. The first canonical axis accounted for $77.67 \%$ of the total variance explained in fungal community structure and were mostly correlated with $\mathrm{WT}$ and $\mathrm{PO}_{4}{ }^{3-}$, while the second canonical axis accounted for $15.35 \%$ of the variance and was mostly correlated with pH. The CCA biplot showed that Basidiomycota and Ascomycota were more related with high temperature. While Chytridiomycota and Cryptomycota with higher levels of $\mathrm{PO}_{4}{ }^{3-}$.

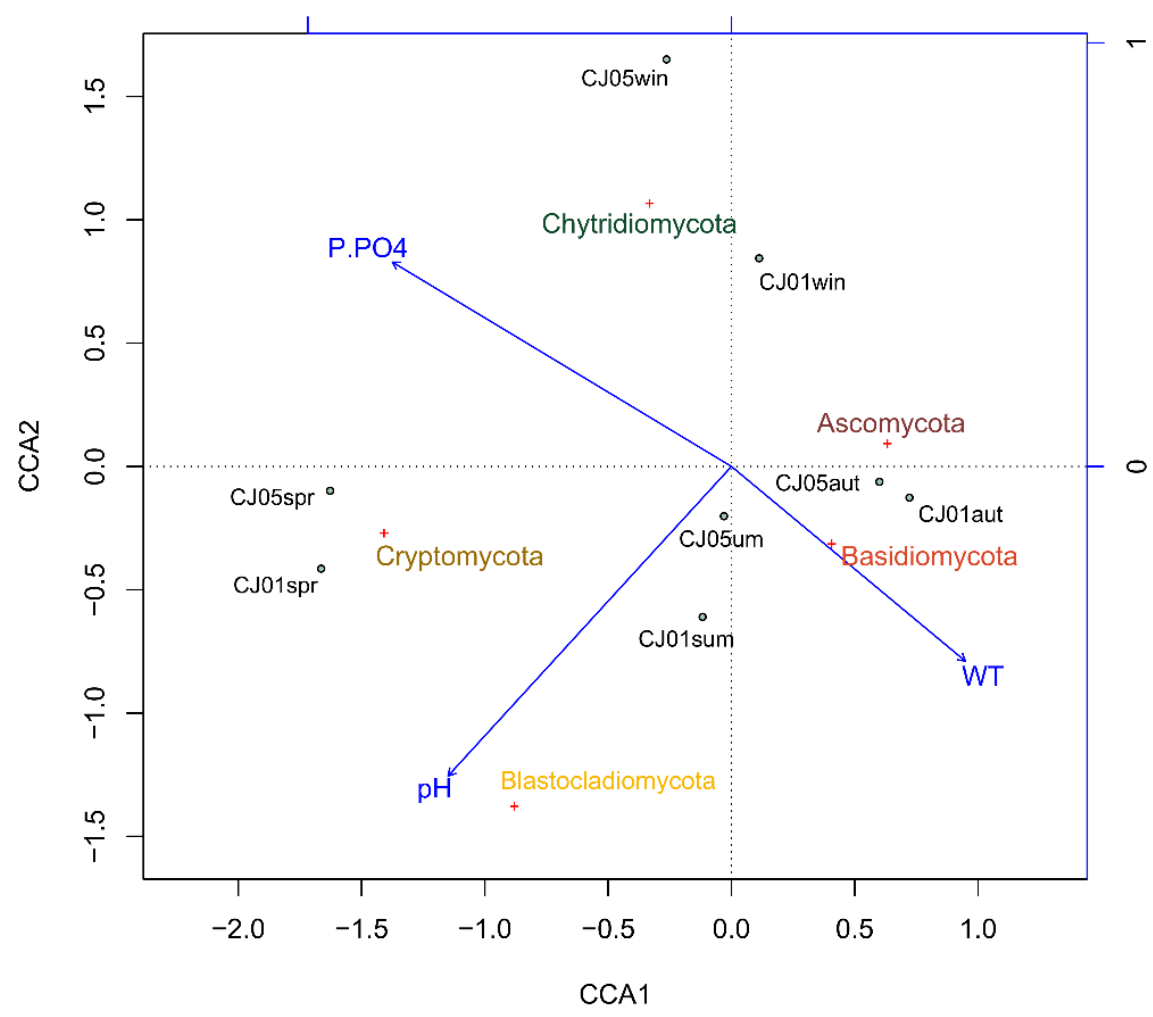

Figure 7. Canonical correspondence analysis (CCA) ordination plot of fungal phylum and environmental parameters. WT: water temperature; P-PO4: orthophosphate

\section{Discussion}

It is well known that various commonly used primers used to study microbial diversity of environmental samples may introduce biases during amplification (Tedersoo et al., 2015). Some researchers prefer the SSU rDNA sequence and others prefer ITS regions to survey fungi from environmental samples (Richards et al., 2012). The ITS approach is useful for determining species diversity, but it is of limited use for extrapolating early diverging fungal lineages and investigating novel groups (Horton and Bnms, 2001), therefore ruling out its use as the only marker for phylogenetic studies (Frenken et al., 2017). For early diverging fungal lineages, sequences from the small subunit (SSU) rRNA gene (18S) can provide the affiliation of higher taxonomic ranks (Cole et al., 2014). Some researchers have focused on sampling the SSU rRNA 
gene to identify novel fungal diversity among higher taxonomic groups (Jebaraj and Raghulnimar, 2009; Preston, et al., 2012; Kivlin and Treseder, 2015), despite that this gene cannot discriminate between closely related fungal species. In some studies, the small subunit has been particularly useful for phylogenetic placing of Chytridiomycota and Cryptomycota and other aquatic fungi (Ishii et al., 2015; Panzer et al., 2015). We pay more attention to the new fungal diversity among higher taxonomic groups, so we prefer the SSU rDNA sequence primers.

The significance of planktonic fungi in the large reservoir is unclear, mainly because data on fungal taxonomy and function in this habitat have been limited. Taxonomically, aquatic hyphomycetes that typically decompose leaf litter and wood are mainly associated with Ascomycota, and only a small percentage are affiliated with Basidiomycota. In this study, Ascomycota and Basidiomycota were the dominant phyla in the warm season, but the relative abundance of these phyla was low in the spring (Fig. 2A). This change may reflect changes in the function of the whole fungal community. For instance, Biatriospora (Ascomycota), Cladosporium (Ascomycota) and Rhodotorula (Basidiomycota) reach their maximum relative abundance in autumn. Biatriospora is associated with plant material found in aquatic habitats (Kolařík et al., 2017). Cladosporium species are agents of decay, deterioration, or a cause of allergies or even plant or animal disease, and often have a high environmental impact (Schubert et al., 2007). Rhodotorula is a saprophytic fungus that can decompose many substances (Wang et al., 2018).

In this study, the relative abundance of Chytridiomycota was the lowest in spring (Fig. 2A). In general, the number of parasitic chytrids increases with the growth of phytoplankton (Ibelings et al., 2004). Compared to winter, the relative abundance of Chytridiomycota was still much lower in spring (Fig. 2A). This phenomenon may be attributed to the dominance of Cryptomycota in spring (more than 60\%). The dominance of Cryptomycota was mainly due to OTU97 (LKM11). Environmental clades LKM11 and Rozella formed the deepest-branching clade of fungi (Lara et al., 2010). Cryptomycota are parasitic fungi, and some Cryptomycota are parasitic in chytrids (Gleason et al., 2014). We hypothesize that OTU97 may be a hyperparasite. Furthermore, the abundance of chytrids may be limited by a large number of Cryptomycota. This conforms to the theoretical assumption that both a decline in phytoplankton populations and an increase in hyperparasite populations as the growing season progresses would result in a decrease in chytrid populations (Gleason et al., 2014). Another possible explanation for the dominance of Cryptomycota in the spring is that some Cryptomycota can parasitize green algae (Held, 1981). In the Three Gorges Reservoir, green algae dominate in spring and reach maximum biomass (Wang et al., 2015). A large number of green algae provide hosts for Cryptomycota so that Cryptomycota can thrive.

In winter, diatom is the dominant group in the Three Gorges Reservoir, its biomass accounts for the highest proportion (Wang et al., 2015). In the Three Gorges Reservoir, the average water temperature is higher than that of air temperature by $10{ }^{\circ} \mathrm{C}$ in winter (Zhou et al., 2019). This higher temperature is conducive to algae growth. In this survey, the relative abundance of Chytridiomycota was the highest in winter. Generally, the dominance of parasitic fungi in winter is unexpected, given the usual lack of suitable algal hosts during this period. Diatoms reach their maximum biomass in winter could explain the dominance of Chytridiomycota with parasitic lifestyles, diatoms provides suitable algal hosts for chytrids. In this study, most taxa of Chytridiomycota belong to 
the parasitic genus Zygorhizidium (80.01\% of total Chytridiomycota reads). Zygorhizidium genus is conducive to the growth of cladoceran zooplankton (Kagami et al., 2007). Thus, chytrid acts as a direct link between inedible or poorly nutritious phytoplankton and filter-feeding zooplankton (Wurzbacher et al., 2010; Haraldsson et al., 2018). This indicates the ecological importance of mycoloop in freshwater ecosystems.

Terrestrial filamentous fungi can be introduced into lakes through spores and pieces of mycelia during inflowing stream, rainwater and wind events (Voronin, 2014). The vegetation on both sides of the Three Gorges Reservoir is dense, and a large number of wood and leaf litter enter the reservoir via flowing streams in the warm season. This litter carries a large number of fungi. In our survey, we found that 18 OTUs only occur in warm seasons. In the Basidiomycota and Ascomycota phyla, terrestrial taxa are the most dominant taxa. For example, OTU187 (Cantharellales) and OTU51 (Cladosporium) are considered terrestrial taxa (El-Nagdy and Nasser, 2000). The relative abundance of these terrestrial fungi varies according to time. This variance may be one of the reasons for the temporal variations in the fungal community. Based on our observations, it can be implied that allochthonous inputs are an important source of fungal species in aquatic ecosystems.

In this survey, we also observed an obvious temporal variation in functional guilds of fungi (Fig. 3). In cold seasons (spring and winter), fungal parasites dominated functional guilds. With an increase in temperature, the proportion of saprotrophic functional guilds also increases. The highest proportion of saprotrophic functional guilds occurs in autumn. There is tremendous leaf fall in autumn, and a lot of organic matter is input into the reservoir. Saprotrophic fungi depend strongly on the organic carbon content across biomes (Fierer et al., 2009). The input of allochthonous organic matter can impact the fungal community in the reservoir. Therefore, saprotrophic fungi increase as a result of increased organic matter inputs. Seasonal allochthonous inputs are likely important drivers of the fungal community in the reservoir. Interestingly, phytoplankton biomass was highest in summer and lower in autumn in the Three Gorges Reservoir (Wang et al., 2015). The increase in pathotroph-saprotroph OTUs in summer and autumn may be related to the presence and senescence of algal taxa during phytoplankton succession along the season (Sun et al., 2017). However, in this study, only 30 OTUs $(30 / 61,49.2 \%)$ were annotated by the FUNGuild annotated database. The FUNGuild database has been cited by a large number of studies (Lu et al., 2018; Toju et al., 2018; Philpott et al., 2018; Jacobsen, et al., 2018). A previous study revealed that fungal guild characteristics were well represented and that guild assignment relies heavily on accurate OTU taxonomic identification (Nguyen et al., 2016). There are a large number of unassigned groups of fungi in our results, and more accurate results need to be further studied.

Chytridiomycota and Cryptomycota were mostly related with higher $\mathrm{PO}_{4}{ }^{3-}$ levels. Fungi react indirectly to physico-chemical factors; this implies that $\mathrm{PO}_{4}{ }^{3-}$ ions may be indirectly affecting these fungi. Furthermore, $\mathrm{PO}_{4}{ }^{3-}$ ion is a limiting nutrient in aquatic systems, and it is especially critical for autotrophs (McMahon and Read, 2013). The presence of phosphorus in lakes is positively correlated with phytoplankton ( $\mathrm{Lv}$ et al., 2011). In this study, some of the recovered Cryptomycota and Chytridiomycota OTUs (e.g. OTU125, Rhizophydiales) are parasites of phytoplankton. These parasites extract nutrients from phytoplankton (Arce Funck et al., 2015). So, plankton can be beneficial for the growth of fungi. Therefore, $\mathrm{PO}_{4}{ }^{3-}$ levels can influence the abundance of fungi. In 
our investigation, we found that the diversity of fungi is reduced by eutrophication. This finding has also been reported by researchers in previous studies (Duarte et al., 2015).

\section{Conclusion}

To conclude, our study showed that the Three Gorges Reservoir region harbors highlevel fungal diversity with an obvious temporal variation of community composition and functional guilds. Parasites thrive in the spring and winter and saprotrophs prevail in the autumn. This highlights a temporal change in the dominant functional role of fungi in the reservoir. Our results show that terrestrial inputs may be an important source of fungal species in reservoir ecosystems. A high species richness and many aquatic hyphomycetes were highlighted, suggesting important ecological roles for fungi in the reservoir region. Finally, to obtain insight into the activity of aquatic fungi and their associated functions, more effective approaches will be necessary in future studies.

Acknowledgments. This work was supported by the National Natural Science Foundation of China (41171045), the Research Fund for Science and Technology Innovation Team of University (T201504). We are thankful to Dr. Yonghong Bi and the Three Gorges reservoir's ecosystem field station for the assistance of sampling. We are grateful to the editor and the anonymous referees for their professional assistance and comments on this manuscript.

Competing interests. The authors declare no competing financial interests.

\section{REFERENCES}

[1] Agha, R., Saebelfeld, M., Manthey, C., Rohrlack, T., Wolinska, J. (2016): Chytrid parasitism facilitates trophic transfer between bloom-forming cyanobacteria and zooplankton (Daphnia). - Scientific Reports 6: 35039.

[2] Arce Funck, J., Bec, A., Perrière, F., Felten, V., Danger, M. (2015): Aquatic hyphomycetes: a potential source of polyunsaturated fatty acids in detritus-based stream food webs. - Fungal Ecology 13: 201-210.

[3] Borneman, J., Hartin, R. J. (2000): PCR primers that amplify fungal rRNA genes from environmental samples. - Applied and Environmental Microbiology 66: 4356-4360.

[4] Canhoto, C., Simões, S., Gonçalves, A. L., Guilhermino, L., Bärlocher, F. (2017): Stream salinization and fungal-mediated leaf decomposition: a microcosm study. Science of the Total Environment 599-600: 1638-1645.

[5] Chen, Z. J., Yuan, J., Sun, F., Zhang, F., Chen, Y., Ding, C. Y., Shi, J. W., Li, Y. Y., Yao, L. G. (2018): Planktonic fungal community structures and their relationship to water quality in the Danjiangkou Reservoir, China. - Scientific Reports 8: 10596.

[6] Cole, J. R., Wang, Q., Fish, J. A., Chai, B., McGarrell, D. M., Sun, Y., Brown, C. T., Porras-Alfaro, A., Kuske, C. R., Tiedje, J. M. (2014): Ribosomal Database Project: data and tools for high throughput rRNA analysis. - Nucleic Acids Research 42: 633-642.

[7] Comeau, A. M., Vincent, W. F., Bernier, L., Lovejoy, C. (2016): Novel chytrid lineages dominate fungal sequences in diverse marine and freshwater habitats. - Scientific Reports 6: 30120.

[8] Countway, P. D., Gast, R. J., Savai, P., Caron, D. A. (2005): Protistan diversity estimates based on 18S rDNA from seawater incubations in the Western North Atlantic. - Journal of Eukaryotic Microbiology 52: 95-106.

[9] Duan, Y. B., Xie, N. D., Song, Z. Q., Ward, C. S., Yung, C. M., Hunt, D. E., Johnson, Z. I., Wang, G. Y. (2018): A high-resolution time series reveals distinct seasonal 
patterns of planktonic fungi at a temperate coastal ocean site (Beaufort, North Carolina, USA). - Applied and Environmental Microbiology 84: e00967-18.

[10] Duarte, S., Barlocher, F., Trabulo, J., Cássio, F., Pascoal, C. (2015): Stream-dwelling fungal decomposer communities along a gradient of eutrophication unraveled by 454 pyrosequencing. - Fungal Diversity 70: 127-148.

[11] Edgar, R. C., Haas, B. J., Clemente, J. C., Quince, C., Knight, R. (2011): UCHIME improves sensitivity and speed of chimera detection. - Bioinformatics 27: 2194-2200.

[12] El-Nagdy, M. A., Nasser, L. A. (2000): Occurrence of zoosporic and terrestrial fungi in accumulated rainfall water in the Riyadh region (Saudi Arabia). - Fungal Diversity 5: 175-183.

[13] Fierer, N., Strickland, M. S., Liptzin, D., Bradford, M. A., Cleveland, C. C. (2009): Global patterns in belowground communities. - Ecology Letters 12: 1238-1249.

[14] Frenken, T., Alacid, E., Berger, S. A., Bourne, E. C., Gerphagnon, M., Grossart, H. P., Gsell, A. S., Ibelings, B. W., Kagami, M., Küpper, F. C., Letcher, P. M., Loyau, A., Miki, T., Nejstgaard, J. C., Rasconi, S., Reñé, A., Rohrlack, T., Rojas-Jimenez, K., Schmeller, D. S., Scholz, B., Seto, K., Sime-Ngando, T., Sukenik, A., Van de Waal, D. B., Van den Wyngaert, S., Van Donk, E., Wolinska, J., Wurzbacher, C., Agha, R. (2017): Integrating chytrid fungal parasites into plankton ecology: research gaps and needs. - Environmental Microbiology 19: 3802-3822.

[15] Frenken, T., Wierenga, J., van Donk, E., Declerck, S. A. J., de Senerpont Domis, L. N., Rohrlack, T., Van de Waal, D. B. (2018): Fungal parasites of a toxic inedible cyanobacterium provide food to zooplankton. - Limnology and Oceanography 63: 2384-2393.

[16] Gao, Z., Johnson, Z. I., Wang, G. Y. (2010): Molecular characterization of the spatial diversity and novel lineages of mycoplankton in Hawaiian coastal waters. - ISME Journal 4: 111-120.

[17] Gerphagnon, M., Agha, R., Martin-Creuzburg, D., Bec, A., Perriere, F., Rad-Menéndez, C., Gachon, C. M. M., Wolinska, J. (2019): Comparison of sterol and fatty acid profiles of chytrids and their hosts reveals trophic upgrading of nutritionally inadequate phytoplankton by fungal parasites. - Environmental Microbiology 21: 949-958.

[18] Gleason, F. H., Lilje, O., Marano, A. V., Sime-Ngando, T., Sullivan, B. K., Kirchmair, M., Neuhauser, S. (2014): Ecological functions of zoosporic hyperparasites. - Frontiers in Microbiology 5: 244.

[19] Gleason, F. H., Scholz, B., Jephcott, T. G., van Ogtrop, F. F., Henderson, L., Lilje, O., Kittelmann, S., Macarthur, D. J. (2017): Key ecological roles for zoosporic true fungi in aquatic habitats. - Microbiology Spectrum 5(2): FUNK-0038-2016.

[20] Grossart, H. P., Rojas-Jimenez, K. (2016): Aquatic fungi, targeting the forgotten in microbial ecology. - Current Opinion in Microbiology 31: 140-145.

[21] Han, L. F., Gao, B., Zhou, H. D., Xu, D. Y., Wei, X., Gao, L. (2015): The spatial distribution, accumulation and potential source of seldom monitored trace elements in sediments of Three Gorges Reservoir, China. - Scientific Reports 5: 16170.

[22] Haraldsson, M., Gerphagnon, M., Bazin, P., Colombet, J., Tecchio, S., Sime-Ngando, T., Niquil, N. (2018): Microbial parasites make cyanobacteria blooms less of a trophic dead end than commonly assumed. - ISME Journal 12: 1008-1020.

[23] Hassett, B. T., Borrego, E. J., Vonnahme, T. R., Rämä, T., Kolomiets, M. V., Gradinger, R. (2019): Arctic marine fungi: biomass, functional genes, and putative ecological roles. - ISME Journal 13: 1484-1496.

[24] Held, A. A. (1981): Rozella and Rozellopsis: naked endoparasitic fungi which dress-up as their hosts. - Botanical Review 47: 451-515.

[25] Horton, T. R., Bnms, T. D. (2001): The molecular revolution in ectomycorrhizal ecology: peeking into the black-box. - Molecular Ecology 10: 1855-71.

[26] Huang, X. F., Chen, W. M., Cai, Q. M. (1999): Survey, Observation and Analysis of Lake Ecology. - China Standard Press, Beijing (in Chinese). 
[27] Ibelings, B. W., De Bruin, A., Kagami, M., Rijkeboer, M., Brehm, M., Van Donk, E. (2004): Host parasite interactions between freshwater phytoplankton and chytrid fungi (Chytridiomycota). - Journal of Phycology 40: 437-453.

[28] Ihaka, R., Gentleman, R. (1996) R: A language for data analysis and graphics. - Journal of Computational and Graphical Statistics 5: 299-314.

[29] Ishii, N., Ishida, S., Kagami, M. (2015): PCR primers for assessing community structure of aquatic fungi including Chytridiomycota and Cryptomycota. - Fungal Ecology 13: 33-43.

[30] Jacobsen, R. M., Sverdrup-Thygeson, A., Kauserud, H., Birkemoe, T. (2018): Revealing hidden insect-fungus interactions; moderately specialized, modular and antinested detritivore networks. - Proceedings of the Royal Society B-Biological Sciences 285: 20172833.

[31] Jebaraj, C. S., Raghulnimar, C. (2009): Anaerobic denitrification in fungi from the coastal marine sediments off Goa, India. - Mycological Research 113: 100-109.

[32] Kagami, M., Elert, E., Ibelings, B. W., Bruin, A., Donk, E. (2007): The parasitic chytrid, Zygorhizidium, facilitates the growth of the cladoceran zooplankter, Daphnia, in cultures of the inedible alga, Asterionella. - Proceedings of the Royal Society B Biological Sciences 274: 1561-1566.

[33] Kagami, M., Miki, T., Takimoto, G. (2014): Mycoloop: chytrids in aquatic food webs. - Frontiers in Microbiology 5: 166.

[34] Kivlin, S. N., Treseder, K. K. (2015): Initial phylogenetic relatedness of saprotrophic fungal communities affects subsequent litter decomposition rates. - Microbial Ecology 69: 748-757.

[35] Kolařík, M., Spakowicz, D. J., Gazis, R., Shaw, J., Kubátová, A., Nováková, A., Chudíčková, M., Forcina, G. C., Kang, K. W., Kelnarová, I., Skaltsas, D., Portero, C. E., Strobel, S. A., Narváez-Trujillo, A. (2017): Biatriospora (Ascomycota: Pleosporales) is an ecologically diverse genus including facultative marine fungi and endophytes with biotechnological potential. - Plant Systematics and Evolution 303: 3550 .

[36] Kolde, R. (2019): Pheatmap, R Package Version 1.0.12 [Internet]. - https://CRAN.Rproject.org/package=pheatmap (updated Jan 4, 2019; cited Jan 4, 2019).

[37] Lara, E., Moreira, D., López-García, P. (2010): The environmental clade LKM11 and Rozella form the deepest branching clade of fungi. - Protist 161: 116-121.

[38] Lefèvre, E., Letcher, P. M., Powell, M. J. (2012): Temporal variation of the small eukaryotic community in two freshwater lakes, emphasis on zoosporic fungi. - Aquatic Microbial Ecology 67: 91-105.

[39] Lepère, C., Domaizon, I., Humbert, J.-F., Hugoni, M., Debroas, D. (2019): Diversity, spatial distribution and activity of fungi in freshwater ecosystems. - Peer J 7: e6247.

[40] Lu, X. M., He, M. Y., Ding, J. Q., Siemann, E. (2018): Latitudinal variation in soil biota: testing the biotic interaction hypothesis with an invasive plant and a native congener. - ISME Journal 12: 2811-2822.

[41] Lv, J., Wu, H. J., Chen, M. Q. (2011): Effects of nitrogen and phosphorus on phytoplankton composition and biomass in 15 subtropical, urban shallow lakes in Wuhan, China. - Limnologica 41: 48-56.

[42] McMahon, K. D., Read, E. K. (2013): Microbial contributions to phosphorus cycling in eutrophic lakes and wastewater. - Annual Review of Microbiology 67: 199-219.

[43] Meng, C. H., Zhao, B. (2007): Study on the trend of eutrophication after impounding in Three Gorges Reservoir. - Journal of Agro-Environment Science 26: 863-867 (in Chinese).

[44] Nguyen, N. H., Song, Z., Bates, S. T., Branco, S., Tedersoo, L., Menke, J., Schilling, J. S., Kennedy, P. G. (2016): FUNGuild: an open annotation tool for parsing fungal community datasets by ecological guild. - Fungal Ecology 20: 241-248. 
[45] Nilsson, R. H., Anslan, S., Bahram, M., Wurzbacher, C., Baldrian, P., Tedersoo, L. (2019): Mycobiome diversity: high-throughput sequencing and identification of fungi. Nature Reviews Microbiology 17: 95-109.

[46] Oksanen, J., Blanchet, F. G., Friendly, M., Kindt, R., Legendre, P., McGlinn, D., Minchin, P. R., O’Hara, R. B., Simpson, G. L., Solymos, P., Stevens, M. H. H., Szoecs, E., Wagner, H. (2016): Vegan, community ecology package. R Package Version 2.4. [Internet] - http//CRAN.R-project.org/package=vegan (updated 2017 Apr 7; cited 2016 Jun 15).

[47] Pachiadaki, M. G., Rédou, V., Beaudoin, D. J., Burgaud, G., Edgcomb, V. P. (2016): Fungal and prokaryotic activities in the marine subsurface biosphere at Peru Margin and Canterbury Basin inferred from RNA-based analyses and microscopy. - Frontiers in Microbiology 7: 846.

[48] Panzer, K., Yilmaz, P., Weiß, M., Reich, L., Richter, M., Wiese, J., Schmaljohann, R., Labes, A., Imhoff, J. F., Glöckner, F. O., Reich, M. (2015): Identification of habitatspecific biomes of aquatic fungal communities using a comprehensive nearly fulllength 18S rRNA dataset enriched with contextual data. - PLoS One 10: e0134377.

[49] Parks, D. H., Tyson, G. W., Hugenholtz, P., Beiko, R. G. (2014): STAMP: statistical analysis of taxonomic and functional profiles. - Bioinformatics 30: 3123-3124.

[50] Philpott, T. J., Barker, J. S., Prescott, C. E., Grayston, S. J. (2018): Limited effects of variable-retention harvesting on fungal communities decomposing fine roots in coastal temperate rainforests. - Applied and Environmental Microbiology 84: e02061-17.

[51] Preston, M. D., Smemo, K. A., McLaughlin, J. W., Basiliko, N. (2012): Peatland microbial communities and decomposition processes in the James Bay Lowlands, Canada. - Frontiers in Microbiology 3: 70.

[52] Rasconi, S., Jobard, M., Sime-Ngando, T. (2011): Parasitic fungi of phytoplankton, ecological roles and implications for microbial food webs. - Aquatic Microbial Ecology 62: 123-137.

[53] Richards, T. A., Jones, M. D. M., Leonard, G., Bass, D. (2012): Marine fungi: their ecology and molecular diversity. - Annual Review of Marine Science 4: 495-522.

[54] Schloss, P. D., Westcott, S. L., Ryabin, T., Hall, J. R., Hartmann, M., Hollister, E. B., Lesniewski, R. A., Oakley, B. B., Parks, D. H., Robinson, C. J., Sahl, J. W., Stres, B., Thallinger, G. G., Van Horn, D. J., Weber, C. F. (2009): Introducing mothur, opensource, platform-independent, community-supported software for describing and comparing microbial communities. - Applied and Environmental Microbiology 75: 7537-7541.

[55] Schubert, K., Groenewald, J. Z., Braun, U., Dijksterhuis, J., Starink, M. S., Hill, C. F., Zalar, P., de Hoog, G. S., Crous, P. W. (2007): Biodiversity in the Cladosporium herbarum complex (Davidiellaceae, Capnodiales), with standardisation of methods for Cladosporium taxonomy and diagnostics. - Studies in Mycology 58: 105-156.

[56] Seena, S., Bärlocher, F., Sobral, O., Gessner, M. O., Dudgeon, D., McKie, B. G., Chauvet, E., Boyero, L., Ferreira, V., Frainer, A., Bruder, A., Matthaei, C. D., Fenoglio, S., Sridhar, K. R., Albariño, R. J., Douglas, M. M., Encalada, A. C., Garcia, E., Ghate, S. D., Giling, D. P., Gonçalves, V., Iwata, T., Landeira-Dabarca, A., McMaster, D., Medeiros, A. O., Naggea, J., Pozo, J., Raposeiro, P. M., Swan, C. M., Tenkiano, N. S. D., Yule, C. M., Graça, M. A. S. (2019): Biodiversity of leaf litter fungi in streams along a latitudinal gradient. - Science of the Total Environment 661: 306-315.

[57] Shearer, C. A., Descals, E., Kohlmeyer, B., Kohlmeyer, J., Marvanová, L., Padgett, D., Porter, D., Raja, H. A., Schmit, J. P., Thorton, H. A., Voglymayr, H. (2007): Fungal biodiversity in aquatic habitats. - Biodiversity and Conservation 16: 49-67.

[58] Sime-Ngando, T., Lefèvre, E., Gleason, F. H. (2011): Hidden diversity among aquatic heterotrophic flagellates, ecological potentials of zoosporic fungi. - Hydrobiologia 659: $5-22$. 
[59] Simon, M., López-García, P., Deschamps, P., Moreira, D., Restoux, G., Bertolino, P., Jardillier, L. (2015): Marked seasonality and high spatial variability of protist communities in shallow freshwater systems. - ISME Journal 9: 1941-1953.

[60] Sun, J. Y., Song, Y., Ma, Z. P., Zhang, H. J., Yang, Z. D., Cai, Z. H., Zhou, J. (2017): Fungal community dynamics during a marine dinoflagellate (Noctiluca scintillans) bloom. - Marine Environmental Research 131: 183-194.

[61] Tedersoo, L., Anslan, S., Bahram, M., Põlme, S., Riit, T., Liiv, I., Kõljalg, U., Kisand, V., Nilsson, R. H., Hildebrand, F., Bork, P., Abarenkov, K. (2015): Shotgun metagenomes and multiple primer pair-barcode combinations of amplicons reveal biases in metabarcoding analyses of fungi. - MycoKeys 10: 1-43.

[62] Tian, W., Zhang, H. Y., Zhao, L., Zhang, F. F., Huang, H. (2017): Phytoplankton diversity effects on community biomass and stability along nutrient gradients in a eutrophic lake. - International Journal of Environmental Research and Public Health 14: 95.

[63] Toju, H., Tanabe, A. S., Sato, H. (2018): Network hubs in root-associated fungal metacommunities. - Microbiome 6: 116.

[64] Van Hannen, E. J., Mooij, W. M., van Agterveld, M. P., Gons, H. J., Laanbroek, H. J. (1999): Detritus-dependent development of the microbial community in an experimental system: qualitative analysis by denaturing gradient gel electrophoresis. Applied and Environmental Microbiology 65: 2478-2484.

[65] Voronin, L. V. (2014): Terrigenous micromycetes in freshwater ecosystems (review). Inland Water Biology 7: 352-356.

[66] Wahl, H. E., Raudabaugh, D. B., Bach, E. M., Bone, T. S., Luttenton, M. R., Cichewicz, R. H., Miller, A. N. (2018): What lies beneath? Fungal diversity at the bottom of Lake Michigan and Lake Superior. - Journal of Great Lakes Research 44: 263-270.

[67] Wang, J. Y., Wang, Z. C., Li, C., Ye, S. W., Lian, Y. X., Liu, J. S., Zhang, T. L., Li, Z. J. (2015): The spatio-temporal dynamics of phytoplankton community structure in the head region of the Three Gorges Reservoir. - Acta Hydrobiologica Sinica 39: 877-884 (in Chinese).

[68] Wang, Q., Garrity, G. M., Tiedje, J. M., Cole, J. R. (2007): Naive Bayesian classifier for rapid assignment of rRNA sequences into the new bacterial taxonomy. - Applied and Environmental Microbiology 73: 5261-5267.

[69] Wang, W., Zhang, D., Wen, H., Wang, Q., Peng, C., Gao, J. (2018): Soil fungal biodiversity and pathogen identification of rotten disease in Aconitum carmichaelii (Fuzi) roots. - PLoS One 13: e0205891.

[70] Wurzbacher, C. M., Bärlocher, F., Grossart, H. P. (2010): Fungi in lake ecosystems. Aquatic Microbial Ecology 59: 125-149.

[71] Wurzbacher, C., Kerr, J., Grossart, H. P. (2011): Aquatic Fungi. - In: Grillo, O. (ed.) The Dynamical Processes of Biodiversity. Case Studies of Evolution and Spatial Distribution., IntechOpen, London.

[72] Zheng, B. H., Zhang, Y., Fu, G., Zhang, H. L. (2006): On the assessment standards for nutrition status in the Three Gorge Reservoir. - Acta Scientiae Circumstantiae 26: 1022-1030 (in Chinese).

[73] Zhou, J. J., Yang, Q., Zhang, M. (2019): Thermal-effect of the upper Yangtze reservoirs and countermeasures. - Journal of Lake Sciences 31: 1-17 (in Chinese). 


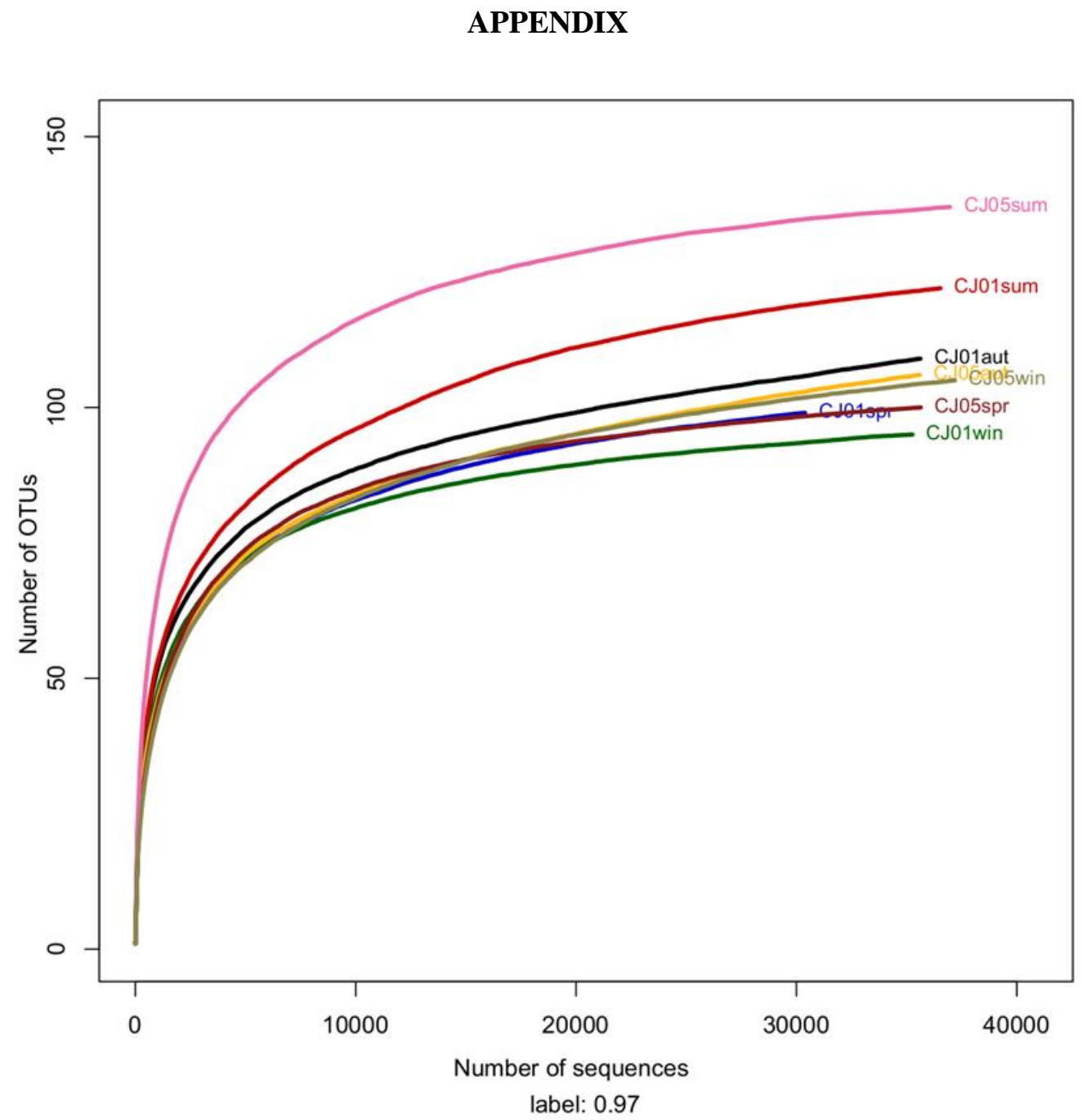

Figure A1. Rarefaction curves. (Spr: spring, sum: summer, aut: autumn, win: winter)
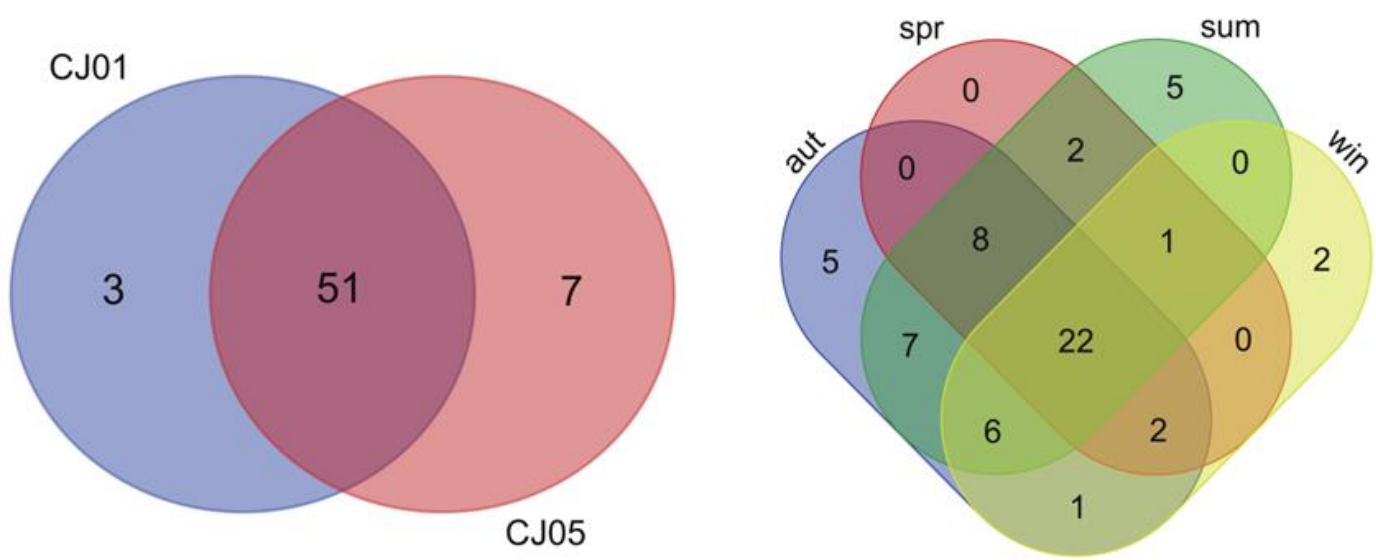

Figure A2. Venn diagrams showing shared and unique seasons and sampling sites by operational taxonomic unit (OTU) 
Table A1. Physical and environmental characteristics of the sampling sites

\begin{tabular}{c|c|c|c|c|c|c|c|c}
\hline & CJ01spr & CJ01sum & CJ01aut & CJ01win & CJ05spr & CJ05sum & CJ05aut & CJ05win \\
\hline TP (g/L) & 0.075 & 0.084 & 0.079 & 0.074 & 0.079 & 0.035 & 0.139 & 0.077 \\
TN (mg/L) & 1.911 & 2.006 & 2.122 & 1.800 & 1.889 & 2.880 & 2.255 & 2.033 \\
COD (mg/L) & 1.143 & 1.451 & 2.222 & 1.569 & 1.219 & 1.725 & 2.343 & 2.000 \\
P-PO4 (mg/L) & 0.076 & 0.054 & 0.051 & 0.064 & 0.076 & 0.050 & 0.054 & 0.075 \\
N-NH4 (mg/L) & 0.027 & 0.026 & 0.069 & 0.094 & 0.006 & 0.039 & 0.028 & 0.072 \\
N-NO3 (mg/L) & 1.667 & 1.378 & 1.149 & 1.718 & 1.635 & 1.327 & 1.184 & 1.787 \\
WT ('C) & 14.800 & 26.820 & 22.280 & 18.440 & 15.000 & 26.570 & 22.210 & 18.410 \\
DO (mg/L) & 9.130 & 6.730 & 7.530 & 9.120 & 10.050 & 6.720 & 7.850 & 9.370 \\
ORP (mV) & 332.400 & 127.000 & 186.000 & 247.000 & 335.500 & 83.000 & 195.000 & 258.000 \\
pH & 8.750 & 8.470 & 8.020 & 8.160 & 8.700 & 8.460 & 8.050 & 7.490 \\
Turbidity (NTU) & 1.200 & 4.500 & 2.100 & 1.500 & 1.400 & 5.800 & 1.900 & 1.400 \\
TLI & 40.692 & 43.703 & 47.433 & 43.144 & 41.488 & 42.429 & 51.414 & 46.178 \\
\hline
\end{tabular}

Spr: spring, sum: summer, aut: autumn, win: winter. WT: water temperature, DO: dissolved oxygen, TN: total nitrogen, P-PO4: orthophosphate, N-NO3: nitrate, TP: total phosphorus, N-NH4: ammonium, COD: chemical oxygen demand, ORP: oxidationreduction potential, TLI: trophic level index

\section{ELECTRONIC APPENDIX}

This manuscript has five electronic appendices with basic data:

Appendix 1. Information on the classes and orders identified from the 8 samples Appendix 2. Compositions of fungal functional guild inferred by FUNGuild Appendix 3. The distribution of fungal functional guild in the phylum level Appendix 4. The distribution of fungal trophic mode in the phylum level Appendix 5. Similarity Percentage analysis (SIMPER) of the 61 fungal OTUs 